\title{
Observer les musées : penser la muséologie au futur
}

observing museums: thinking museology in the future

\section{Olivia Guiragossian}

\section{Q OpenEdition}

\section{Journals}

Édition électronique

URL : http://journals.openedition.org/iss/2124

DOI : $10.4000 /$ iss. 2124

ISSN : 2306-4161

\section{Éditeur}

ICOM - International Council of Museums

\section{Édition imprimée}

Date de publication : 1 août 2020

Pagination : 147-163

ISBN : 978-2-491997-11-3

ISSN : 2309-1290

\section{Référence électronique}

Olivia Guiragossian, «Observer les musées : penser la muséologie au futur », ICOFOM Study Series [En ligne], 48-1 | 2020, mis en ligne le 01 août 2020, consulté le 08 août 2020. URL : http:// journals.openedition.org/iss/2124; DOI : https://doi.org/10.4000/iss.2124 


\section{Observer les \\ musées : penser au futur}

\section{Olivia Guiragossian}

Université Sorbonne-Nouvelle Paris 3, CERLIS Paris, France

RÉSUMÉ

Dans un débat où la question des formes futures de la muséologie émerge, les observatoires de musées constituent un angle d'approche pertinent, s'inscrivant dans une perspective dynamique et évolutive, à la jonction des préoccupations passées, présentes et futures du champ muséal. Force est de constater que ce champ d'étude est relativement invisible et que la vision de la muséologie comme étude de la relation de l'Homme avec son environnement semble réfractaire à une approche numéraire et chiffrée. Cette absence de réflexion sur les méthodes de l'observation fait écho à un manque de connaissance du champ muséal. Les statistiques connues sur les musées, généralement étudiés sous l'angle de la fréquentation des publics, ne reflètent en l'état actuel ni la complexité du monde muséal, ni son évolution dynamique, rarement son contexte environnemental. En nous interrogeant sur la production de connaissances sur le musée - mais hors de lui - nous en esquisserons ici les principaux enjeux afin d'appréhender de potentielles pistes

d'évolutions pour la muséologie.

Mots-clés : Observatoires, musées, muséologie, méthodologie, définition, futur, population, public, data 


\section{Observing museums: thinking museology in the future}

In a debate in which the question of future forms of museology is emerging, museum observatories constitute a relevant approach, from a dynamic and evolving perspective, at the junction of past, present and future concerns of the museum field. It must be noted that this field of study is relatively invisible and that the vision of museology as a study of the relationship between Man and his environment seems refractory to a quantified approach. This lack of reflection on observation methods reflects a lack of knowledge of the museum field. Known statistics on museums - generally studied in terms of audience attendance - do not reflect the current state of the museum world, its dynamic evolution and rarely its environmental context. By asking ourselves about the production of knowledge about the museum - but outside it - we will sketch out here the main challenges in order to understand potential avenues of development for museology

Keywords: Observatories, museums, museology, methodology, definition, future, population, public, data

Comment envisager le futur de la muséologie ? Aussi « relativement récente » que l'institution qu'elle se donne pour objet d'étude (Pomian, 1987), cette discipline est le fruit de siècles d'évolution d'une réflexion sur les pratiques et le rôle des musées, adossée à une transformation effective du champ muséal (Desvallées \& Mairesse, 2005). Aujourd'hui, ce ne sont plus les musées qu'elle investit, mais les universités et les forums académiques internationaux (Brulon Soares, 2015), cristallisant le fossé entre théorie et pratique, entre monde scientifique et monde professionnel. Et dans un sens, si la conception de la muséologie comme discipline scientifique a fait - et fait encore - débat (Deloche, 2015), face aux risques grandissants d'une rupture entre muséologie et musée, c'est aujourd'hui tout le processus de production de connaissances qui doit être remis en perspective.

Dans ce cadre particulièrement porteur pour les questions de méthodologie, les observatoires de musées constituent un espace de recherche pertinent, s'inscrivant dans une perspective dynamique et évolutive, à la jonction des préoccupations passées, présentes et futures du champ muséal. Ce domaine d'étude est pourtant relativement invisible, peu maitrisé du moins par les chercheurs en muséologie. D’une part parce que les compétences mobilisées s'éloignent des formations universitaires traditionnelles, mais aussi car la vision de la muséo- 
logie comme étude de la relation de l'Homme avec son environnement semble réfractaire à une approche numéraire et chiffrée. Les conséquences sont fondamentales et cette absence de réflexion sur les méthodes de l'observation fait écho à un manque flagrant de connaissance du champ muséal. Les statistiques connues sur les musées - généralement étudiés sous l'angle de la fréquentation des publics, mettant en avant une focale centrée sur les musées « superstars » (Frey \& Meier, 2006) - ne reflètent en l'état actuel ni la complexité du monde muséal, ni son évolution dynamique, rarement son contexte environnemental. Elles posent finalement la question de l'appréhension - fractionnée - que nous avons du phénomène muséal. Peut-on réellement se contenter de cette vision partielle pour le comprendre?

Il est nécessaire alors de s'interroger plus précisément sur la manière dont ces savoirs et ces données sont construites. Les observatoires, instruments d'analyse et d'interprétation au cour des sciences sociales, constituent des dispositifs ambivalents, au croisement de l'élaboration des connaissances et des représentations d'une discipline (Le Marec \& Belaën, 20I2). En premier lieu, leur existence est justifiée par la nécessité de mettre en place des outils, des indicateurs, des tableaux de bords, des concepts, offrant une "photographie » du monde observé, et permettant de suivre la mise en ouvre d'une politique - quel que soit son niveau territorial - afin d'en évaluer les performances. Il s'agit donc d'un dispositif de représentation des enjeux économiques, politiques et sociaux des actions menées sur les territoires pour les pouvoirs publics, et donc primordial pour les autorités décisionnaires. Cependant, dans le même temps, les observatoires créent des savoirs et intègrent des problématiques liées à la diversité d'intérêt de la connaissance et aux modes de production des savoirs; dans cet espace de recherche, leur existence n'est pas neutre et contribue à la normalisation et la hiérarchisation de cette diversité (Le Marec \& Belaën, 20I2). Appliqués à l'objet «musée », ils produisent ainsi des données le concernant, en définissant des populations précises, en circonscrivant des enjeux qui seraient majeurs pour leur compréhension et leur fonctionnement. Ils forment de fait des espaces de recherche liant musées et société.

Il est bien difficile pourtant de définir un ensemble complet d'observatoires de musées car rares sont les organismes qui se définissent comme tel. Certains dépendent des instances gouvernementales, d'autres d'universités, d'autres sont encore des bureaux indépendants, des associations ou des centres de coopération. Ces observatoires se constituent ainsi en une nébuleuse de lieux qui observent une ou plusieurs variables des pratiques culturelles. Et bien souvent, les musées sont intégrés dans une plus large étude des statistiques culturelles, constituant l'un des axes prioritaires des politiques européennes (Nuere, 20II). Cela nécessite donc d'identifier la spécificité de la statistique muséale, fondée sur une institution complexe et son expérience de visite, qui n'engendre pas les mêmes problématiques que les industries culturelles, basées sur la reproductibilité en masse de façon standardisée, des œuvres de l'esprit (Guibert, Rebillard \& Rochelandet, 2016). Sous l'hétérogénéité des formats, 
nous retrouvons les activités qui les caractérisent : la définition d'un objet d'étude, la méthodologie de production des données, par le biais souvent de questionnaires auto-administrés et d'enquêtes de terrain, la formalisation et la diffusion des résultats .

En nous interrogeant sur la manière dont les observatoires se sont emparés et s'emparent encore des problématiques du champ muséal, un ensemble de questions émergent : comment les observatoires et leurs problématiques nous permettent-ils de développer et de renouveler l'approche de la muséologie? Quels sont leurs enjeux dans le domaine des musées et quel champ d'action cela dessine-t-il pour le futur de la recherche? Dans un premier temps, nous tenterons de voir comment les observatoires participent de la définition d'une population muséale, dans une approche constructiviste. Cette étape est essentielle pour mettre en exergue les questions de diversité au sein du monde des musées, contribuant de fait à l'élaboration d'une représentation d'un champ de recherche, souvent donné comme acquis, rarement remis en question. Nous étudierons par la suite les évolutions possibles dans le champ de l'observation, au travers des indicateurs et des méthodes, investies par les tendances de la participation, de la collaboration et l'apparition des données massives (Big data), afin de comprendre comment la muséologie peut s'emparer de ces changements pressants, sociaux et technologiques, qui touchent le monde de la statistique. A travers le prisme de l'observation, et sans prétendre recenser toutes les méthodes et outils existants, nous souhaitons ainsi faire émerger quelques problématiques qui contribueront au développement de la muséologie dans - et pour - le futur.

\section{Définir le musée : une « population » à analyser}

Observer donc, mais observer quoi ? Le musée peut être assimilé à une véritable population et sa définition va former le soubassement principal des études d'observation ${ }^{2}$. Il ne s'agit cependant pas d'en extraire l'essence, le rôle et les fonctions, mais bien un ensemble de critères caractéristiques de cette forme «non stable» (Desvallées \& Mairesse, 2005). La problématique des contours du musée est une véritable question, assez peu soulevée, sinon mentionnée, mais rarement abordée dans toute sa complexité, notamment au regard des recensements de musées et de l'évolution de l'institution. Au-delà de son application,

\footnotetext{
I. Nous prenons ici en considération essentiellement des organismes ou institutions qui produisent des données spécifiquement sur les musées et émettent des outils de communication pouvant les restituer au sein de l'espace public et politique. Cette approche se développe non seulement à l'échelle de la France, mais également internationale afin d'établir des points de comparaisons parlants.

2. Cette notion de «population » s'applique d'abord dans le champ de la statistique, en tant qu'ensemble d'éléments caractérisés par un critère permettant de les identifier sans ambiguité. Néanmoins le terme peut également être emprunté à la démographie : si cette idée peut être discutée au regard du musée, qui n'est pas une entité biologique mais une production de l'Homme, elle peut se justifier par la création de différents groupes au sein d'une population, le dynamisme de ces groupes et leur ancrage territorial et territorial qui nécessitent d'en étudier le développement.
} 
cette définition du musée pose la question bien plus épineuse des contours du champ muséal, dont les limites sont mouvantes. Par ce biais, transparaît la question centrale de la reconnaissance de la diversité des musées dans le monde.

\section{Ceci n'est pas un musée}

Depuis des décennies, le musée est régulièrement enclin à un processus de déf1nition et de redéfinition, qui témoigne de son adaptation à son environnement (Goode, I895). Pourtant celui-ci n'est ni anodin, ni aisé à mettre en place. Il est certainement plus simple de définir le musée « en négatif - par ce que l'on considère qu'il n'est pas ou ne doit pas être - ou par cercles concentriques - par ce que l'on considère qu'il peut éventuellement être, doit être, pourrait être » (Botte, Doyen \& Uzlyte, 20I7, p.I7) que d'en établir une définition concise et déterminée. Toutes les définitions existantes, mises en place par les Etats ou les associations de professionnels - la plus répandue restant à ce jour celle donnée en 2007 par le Conseil International des Musées (ICOM) - en soulignent la difficulté, tant cet objet est complexe, multiforme et multifonctionnel. Pourtant les enjeux d'un renouvellement de définition sont réels et palpables, ils s'étalent sur des problématiques de législation - et par extension de financement, de géopolitique ou encore de société. La plupart des pays ont établi par le biais de leurs textes législatifs ou de leurs organisations internationales des définitions du musées (Mairesse \& Desvallées, 2005), répondant ainsi à cette question un peu naïve : "qu'est-ce qu'un musée ?". Car finalement, quand nous parlons de l'augmentation spectaculaire des institutions muséales dans le monde : 22 ooo musées en 1975, 49 ooo en 2004 et «plus de 55 ooo » de nos jours (De Gruyter Saur, 2019), de quoi s'agit-il réellement? Ces chiffres sont-ils même justifiés et plausibles?

D'un point de vue historique, la préoccupation d'un recensement des musées n'est pas récente et permet une relecture pertinente de l'évolution des institutions muséales au cours des deux derniers siècles. On compte moins d'une trentaine d'établissements proches de la forme moderne du musée à la veille de l'ouverture du Musée du Louvre (I793), principalement en Europe (Pommier, 1995). Au XIXe siècle, en France, on peut étudier le développement des institutions muséales sous quatre périodes :

[...] vers 1815 , ils étaient une trentaine; vers 1870 , environ 200 ; vers 1890 ; environ 300 ; vers 1914, environ 550 . (Pomian, 1994, p. 356)

99

Ces chiffres, approximatifs, issus de plusieurs rapports ${ }^{\mathrm{T}}$ sont évidemment à relativiser. Comme l'indique Krzysztof Pomian, ils dépendent de la définition

I. Krzysztof Pomian mentionne notamment les rapports suivants :

Lapause H. (I908). Rapport présenté au nom de la Commission /chargée d'étudier toutes les questions rela- 
du musée alors considérée. Ils permettent néanmoins de dégager une tendance, montrant qu'en France, la seconde moitié du XIXe siècle apparaît comme la «grande époque de la multiplication des musées ». Déjà, à l'échelle internationale, les comparaisons sont délicates, avec l'Empire allemand dont on connait le nombre de collections publiques (220 vers I88o) ou la Grande Bretagne (240 musées vers la fin des années i880) (Pomian, 1994). Plus développés et systématisés, ces enjeux existent toujours aujourd'hui.

En France, actuellement, la majorité des statistiques muséales sont produites par le Ministère de la Culture, autour de deux services (le Département de la Politique des Publics, travaillant sur la base Muséofile et sur l'Observatoire des publics créé en 1989 ; le Département des Etudes de la Prospective et des Statistiques). S'il fait état aujourd'hui de I219 musées de France, la population étudiée est restreinte aux institutions conformes à la loi du 4 janvier $2002^{1}$, qui supposent pour être ainsi accréditées la présence d'une collection et la mise en place d'un inventaire ; la mise en place d'un projet scientifique et culturel ; un engagement sur les missions de conservation, d'acquisition, de d'étude ; la présence d'un personnel scientifique et la présence d'un service éducatif. Cet ensemble de conditions, donnant lieu à une reconnaissance gouvernementale, dessine les contours d'une population singulière.

Sur cet échantillon - dont on se rend bien compte qu'il ne permet qu'une approche partielle des musées en France, des questions techniques se posent. Il ne suffit pas de donner une liste de conditions, mais de penser la définition de la population muséale en termes d'opérationnalité. Doit-on prendre par exemple en compte le musée comme unité administrative ? Ou comme un site, avec une adresse définie ? Le cas du Musée des Arts Décoratifs (MAD) est significatif : composé de trois entités (Musée des Arts Décoratifs, Musée de la Mode et du Textile, Musée de la Publicité) mais seulement d’un site, on en retrouve trois entrées distinctes dans la liste des Musées de France. Autre exemple : que signifie la permanence d'une collection? Un musée qui vient d'ouvrir ne peut prouver la permanence de son existence, de même que la longévité d'un musée ne permet pas d'établir sa permanence dans le futur (Waltz, 2017). De même, à partir de quel volume horaire ou de journées d'ouverture peut-on considérer qu'une institution est « ouverte au public » ? Ces questions

tives à lorganisation des musées de province et à la conservation de leurs richesses artistiques], Paris;

De Chennevières P. (I878). Rapport adressé à M. le Ministre de l'Instruction publique, des Cultes et des Beaux-Arts sur l'administration des arts, Paris ;

Guédy T. (I889). Musées de France et collections particulières, Paris ;

Georgel C. (1994), De l'art et de la manière d'enrichir les collections. Dans C. Georgel (r994). La jeunesse des musées: les musées de France au XIXe siècle. Paris: Réunion des musées nationaux.

I. D'après la loi no $2002-5$ du 4 janvier 2002 relative aux musées de France, «L'appellation «musée de France » peut être accordée aux musées appartenant à l'Etat, à une autre personne morale de droit public ou à une personne morale de droit privé à but non lucratif. Est considéré comme musée, au sens de la présente loi, toute collection permanente composée de biens dont la conservation et la présentation revêtent un intérêt public et organisée en vue de la connaissance, de l'éducation et du plaisir du public. » 
se posent sur un ensemble de critères : unité d'observation, profil économique, indépendance financière et subventions de l'Etat, notion de permanence, rôle social du musée, accès au public, activité d'acquisition. Il existe donc une étape de questionnements nécessaire pour bien comprendre les ressors du recensement des musées et la mise en place de critères définissant une population : celle de la conceptualisation et de la mise en opération de cette définition.

Dans l'optique d'une approche internationale, si ce travail n'est pas fait en amont, il ne pourra s'agir que d'un travail d'harmonisation des données. La dynamique à l'œuvre aujourd'hui dépasse pourtant la compilation des données nationales et porte sur la mise en place d'une grille d'analyse commune, prenant en compte ce travail d'opérationnalisation des définitions et des spécificités des législations nationales dans lequel elles sont développées. C'est le cas pour EGMUS (European Groups on Museums Statistics) qui a amorcé des réflexions préliminaires lors de sa réunion annuelle à Bucarest (2019), mais également du programme Ibermuseos mis en place par l'Observatoire des Musées Ibéro-Américains dans le cadre du projet de Registre des musées ibéro-américains (2013). Dans ce dernier cas, par un procédé de restriction des critères, les populations muséales prises en compte sont modifiées, plus restreintes, à l'échelle transnationale. A titre d'exemple, on ne compte dans ce registre que 245 musées pour l'Argentine alors que le gouvernement en déclare Ior7, ou 55 pour le Pérou qui en annonçait initialement 37r. Dans les deux cas, il devient évident que la définition de dénominateurs communs s'inscrit dans un processus de négociations, nécessitant une connaissance aguerrie du secteur muséal. A plusieurs titres, le musée ne s'envisage donc plus comme une entité abstraite, mais comme un ensemble de populations, renouvelant ainsi les approches possibles du champ muséal.

\section{Métamorphoses et mutations : temps des musées, temporalité de la recherche}

Les musées naissent, vivent et meurent. Parfois ils se transforment, se métamorphosent, et ces mutations ne sont pas sans impact sur le champ muséal. Comment envisager cette évolution dans les instruments d'observation? L'observatoire s'inscrit dans un rapport temporel complexe : le temps de l'observation doit être ramené au temps d'existence de l'objet d'étude. Cette approche dynamique est éloquente au regard du processus de définition du musée. S’il est considéré en premier lieu comme une "institution permanente ", il ne cesse pourtant de se construire et de se transformer, objet de multiples évolutions et mutations qui s'opèrent en fonction des changements de la société et des besoins de l'Homme. De manière effective, les musées peuvent « changer de nom, de lieu, de territoire de référence et seules demeurent les collections qui sont soumises à une relecture complète » (Chevallier, 20I3, p. II) mais ils peuvent également changer de projet muséal, en instaurant une relecture de leurs espaces. 
Cette question de la temporalité influe non seulement sur le temps de l'observation, mais pose la question du choix du format de présentation des données, car une base de données figée, même réactualisée, ne fournira forcément pas les mêmes informations qu'une base de données évolutive, intégrant la notion de changement. La base Muséofile, produite par le Service des Musées de France, est assez significative de cette problématique : la première version - sous forme d'une liste des Musées de France, auxquels sont associés une fiche technique, et d'une cartographie - n'a pas été actualisée jusqu'en 20I8. On pouvait alors y trouver alors sur les jo entrées concernant la ville de Paris, 5 musées fermés (soit définitivement ou relocalisés comme le Musée national des Traditions Populaires dont les collections ont été transférées au MuCEM) et II institutions ne bénéficiant pas - ou plus - de l'appellation « Musée de France », soit un taux d'obsolescence des données de $22 \%$. Si le projet de la base est actuellement en cours d'actualisation, on peut s'interroger sur sa pertinence : va-t-on garder la mémoire de ces modifications? Comment les retranscrire? Que disent-elles de l'évolution du domaine muséal?

Le temps de la recherche est un temps long, qui semble parfois contraire à l'évolution des musées. Il existe par ailleurs un biais technologique non négligeable dans cette approche : il est plus facile d'actualiser des données numériques, que des répertoires ou des guides touristiques. Cependant si ceux-ci conservent pendant longtemps la trace de l'obsolescence des données, ils permettent de conserver les traces passées de cette histoire des musées. Une histoire parfois faite de modifications et de fermetures, qui peut permettre d'obtenir une vision en négatif du monde des musées. De manière générale, cette dynamique du changement va nécessiter un outil évolutif, intégrant cette notion de métamorphose pour permettre une compréhension globale du phénomène muséal.

\section{La diversité muséale en question}

Que ce soit au sein ou à l'extérieur de la population muséale définie par les observatoires, la mise en place de catégories caractérisant les musées, leur forme et leurs thématiques, est certainement un enjeu primordial, produisant une vision de la diversité muséale. Par extension, les catégories de musées investissent à la fois les problématiques inhérentes au projet muséal, de la communication de leur existence auprès des publics aux comportements de visites supposés. Ce n'est pas une nouveauté : il n'existe actuellement pas de consensus concernant ces typologies. Si la classification de George-Henri Rivière (musées d'art, musées des sciences de l'Homme, musées des sciences de la nature, musées des sciences et des techniques, musées multidisciplinaire et interdisciplinaire) est reprise par Gob et Drouguet (2010), il existe des classements différents, présentés par Ambrose et Paine (2012), Dean et Edson (1994), ou encore Zubiaur Carreño (2004). A la jonction de l'histoire des sciences et des savoirs et des pratiques muséales, ces typologies permettent de créer des regroupements mentaux et d'affiner les mouvements des populations muséales. 
Ce qui nous intéresse ici, c'est le fait que le périmètre de l'observation forme des espaces de vide qui délaissent les expériences muséales sortant des cadres institutionnalisés, ce qui a évidemment un impact sur une approche mondiale du phénomène muséal. Réinterrogeant les critères du musée, de nouvelles catégories émergent, formant des populations « déviantes». En première ligne, l'aspect central de la collection invisibilise les formes du musée qui n'en possèdent pas. Rappelons que, précurseur dans le champ de la Nouvelle Muséologie, l'écomusée, qui témoignait d'un intérêt sans faille pour le lien communautaire et territorial (Rivière, I989) ne nécessitait aucune collection préalable à sa création. Le concept développé à partir de 1974 était celui d'un musée-territoire, constitué par un ensemble d'antennes, de parcours au sein du territoire, de manière théorique, d'un musée véritablement lié au fonctionnement de sa communauté : le musée pensé pour et par la population. Et pourtant, les écomusées font partis de ces formes nouvelles du musée qui ont souffert d'un déficit de reconnaissance à leur création, puisqu“ « un musée sans collection n'est pas un musée » (Debary, 2002). Pourtant ce modèle s'est développé pendant plus de 50 ans dans le monde (De Varine, 2017). Aujourd'hui encore, le programme gouvernemental lancé par le Brésil sur les Pontos de Memoria (OEI \& IBRAM, 20I6) conçu comme un ensemble d'actions et d'initiatives de reconnaissance et de valorisation de la mémoire sociale, fait écho à cette logique. Ainsi, se déploie une dimension humaine et vivante au sein de l'institution muséale. Les musées de la personne, les centres d'interprétation ou mêmes les centres de sciences, ne mériteraient-ils pas d'être intégrés au sein de la population muséale? Quelle place donner aux musées virtuels? Quid également des musées asiatiques où les musées peuvent se concevoir sans collections (Morishita, 2010) ?

Déconstruire pour mieux comprendre : réinterroger les données existantes sur les musées, fournies par les observatoires, et leur processus de production, est une étape essentielle aujourd'hui pour comprendre leur signification, intégrer le champ de la muséologie et ouvrir un champ des possibles pour nos connaissances sur les musées. En appliquant spécifiquement l'idée que le musée peut se construire comme une population profondément diversifiée, l'étude du champ muséal cristallise des lignes de force mais également de faiblesses. De manière irrémédiable, l'établissement de critères pour définir une population à étudier suppose d'en invisibiliser une partie, mais ce processus est nécessaire à bien des égards pour construire une vision globale du phénomène muséal à l'échelle mondiale.

\section{Le renouvellement de l'observation : participation, collaboration, data}

À la jonction entre le musée comme institution et l'observatoire comme espace de recherche, les transformations de la société interrogent à leur tour les comportements étudiés dans le champ muséal. Si l'on a écrit sur les modifications qui traversent le musée et ses pratiques, l'émergence de nouvelles tendances dans le monde de la recherche statistique, issues des mutations sociales et tech- 
nologiques, s'applique à la production de données sur le musée et le muséal. La manière de penser la recherche - au niveau des indicateurs et des techniques de l'observation - et de la diffuser, sont destinées à évoluer. Par exemple, que ce soit l'apparition des Big data (Trendswatch 20I4) et de l'Open data (Trendswatch 2015), elles sont prises en compte dans les évolutions possibles du muséal définies par le Center For the Future of Museums. Ces tendances - participation, collaboration, intégration des données massives - ne sont évidemment pas spécifiques au champ muséal, mais elles apparaissent avec force dans le champ d'action des observatoires, donnant lieu à des nouvelles politiques et stratégies. Modifiant le paradigme de l'accès aux données, elles ouvrent des perspectives renouvelées quant à leur production, leur exploitation et leur réutilisation, dans le domaine de la muséologie.

\section{Du public comme indicateur au public-acteur}

La grande majorité des statistiques muséales en France et dans le monde portent sur la fréquentation des musées, mettant en évidence la place du public au cœur du projet scientifique, dans une dynamique amorcée par le courant de la Nouvelle Muséologie dans les années i980. Ainsi, qu'il s'agisse d'observer les variations de la fréquentation du nombre de visiteur à l'échelle d'une institution ou d'un territoire ou de l'analyse de leur diversité sociale, les études de publics sont devenues le principal indicateur de la vitalité du musée et un argument de légitimité auprès des autorités décisionnaires (Sepulveda-Koptche, 20I0). Le choix des indicateurs dans les méthodes de l'observation n'est pas neutre : cette focale sur les publics s'inscrit dans la mise en place d'une politique affirmée de démocratisation culturelle Pouvoir appréhender les pratiques de visite permet ainsi d'établir une politique construite des publics et d'élaborer ou de renforcer un projet culturel cohérent (Lehalle \& Mironer, 1993) : le musée est ainsi perçu sous l'angle de la pratique des visiteurs, qui deviennent l'unité principale de mesure.

Il s'opère cependant un basculement dans cette perception du public : il ne représente plus une entité figée dans le temps seulement juxtaposée aux préoccupations des musées. Dans un mouvement de rassemblement et d'intégration de la population au sein des institutions muséales apparaît une injonction à la participation qui constitue une tendance générale à la fois de la société, du secteur culturel et ici, plus particulièrement des musées (Eidelman, 20I7). De manière générale, que ce soit au travers des budgets participatifs, du financement participatif ou encore des expositions participatives, ce ne sont plus les professionnels et les chercheurs qui définissent les tendances, mais les citoyens mobilisés, se sentant a minima concernés par les sujets traités. Les problématiques de la participation investissent ainsi donc le musée où les visiteurs souhaitent avoir la capacité de « discuter, partager, remixer ce qu'ils consomment » (Simon, 20Io, p. ii). Cette problématique est sensible au niveau de la production des connaissances sur le musée : les rapports sur le futur de l'institution intègrent de plus en plus fréquemment des consultations citoyennes, 
notamment au travers de plateformes numériques. Les indicateurs, mais également les pratiques de l'observation, se modifient et la volonté d'une efficacité au niveau des politiques publiques et des politiques culturelles interroge avec force ce que veut le visiteur/le spectateur/le consommateur.

Les rapports Museums 2020 publié par la Museums Association (2012) et le Rapport Musée du XXI siécle dirigé par Jacqueline Eidelman (20I7) sont témoins de cette dimension. Pour cette dernière, la plateforme collaborative "Imaginons ensemble le musée du XXI ${ }^{e}$ siècle » a été ouverte au public pendant 6 semaines (26 septembre - 15 novembre 2015), recensant Io ooo visites, I 057 contributions et $454 \mathrm{I}$ votes autour de trois questions phare : les raisons de la visite, les attentes et l'implication dans la vie du musée. L'analyse lexicale du contenu et des votes - réalisée par le Credoc a permis de mettre en avant quatre thèmes majeurs : l'accessibilité et la gratuité ; la transmission de valeurs et la vocation sociale; le renouvellement de l'expérience de visite et enfin, le numérique. Dans la même logique, mais sur un sujet plus normatif, s'interrogeant sur les contours et la définition du musée pour le XXIe siècle, l'ICOM et son Comité pour la Définition du Musée, Perspectives et Potentiels (MDPP) ont enrichi leur méthode de travail (traditionnellement par tables-rondes et conférences) par une plateforme numérique, ouverte en janvier 2019 afin de permettre à tous (professionnels ou non) de proposer sa propre définition du musée : 269 contributions ont été recueillies, illustrant les préoccupations de la communauté muséale et citoyenne. Les modifications sont profondes : l'usager, et par extension le citoyen, devient donc acteur dans l'observation et la prédiction de tendances culturelles.

Cependant, cette approche - si elle est toujours adossée à un travail d'observation «traditionnel »- rencontre des limites en termes de légitimité et de représentativité. Dans le cadre notamment du rapport de la mission Musées du $X X I^{e}$ siècle, une sociologie sommaire esquissait un portrait des contributeurs. Loin d'illustrer une ouverture sociale, ceux-ci étaient en grande majorité déjà proches de la culture, familiers des musées ou jeunes professionnels. Cette question est également apparue au sujet des contributions pour une nouvelle définition du musée, dont on ne sait si elles émanaient de professionnels ou de néophytes - puisque qu'anonymes : «tout le monde » est-il habilité à contribuer sur ces thématiques aux enjeux forts?

\section{L'approche contributive appliquée aux données}

Cette approche contributive et participative est loin de se limiter aux attentes exprimées par le public. Si, pendant longtemps, la mesure statistique a été considérée comme une question de spécialistes (Cardon, 2015), les frontières aujourd'hui sont bouleversées. L'introduction des data sciences dans les pratiques sociales, et dans la vie des institutions muséales, soulève la question de la nature des données et de l'identité des « pourvoyeurs de données ». La logique des bases de données informatiques et leurs applications aux méthodes de classement des objets avaient déjà investi le champ de la muséologie dès les années i960 
(Mairesse, 2016, p. 20). Cette question mérite maintenant d'être soulevée sous l'angle de la production de la connaissance impliquée par l'émergence des datas qui s'intègrent dans un mouvement de société global pour le futur. Parler d'open data (données ouvertes), c'est faire référence à des donnés primaires, pouvant être collectées par les organismes publics ou privés et devenant accessibles et réutilisables sans restriction. Cette politique constitue ainsi un bouleversement à la fois culturel et cognitif, s'inscrivant au cour de la transformation numérique des sociétés. En France, la Loi pour une République numérique (2016) vise à garantir l'accès de tous aux opportunités liées au numérique. Parmi les principes définis se trouve l'ouverture par défaut (ou par principe) de l'ensemble des données produites ou collectées; le principe de gratuité ; l'utilisation de standards ouverts et facilement réutilisables et enfin la création du service public de la donnée.

Historiquement utilisée pour rendre accessibles les ressources des musées - de l'initiative du Rijksmuseum d'Amsterdam en 201 au Digital Catalog Online des musées en 2019 - la collaboration est envisagé à toutes les échelles du partage des données. Le rapport Ouverture et partage des données publiques culturelles, pour une (r)évolution numérique dans le secteur culturel publié en France par le Ministère de la Culture (2013) souligne à ce titre le rôle déterminant des institutions du secteur culturel dans la production de données, dites de qualité et labellisées scientifiquement. Un mouvement est alors à l'œuvre, notamment dans le cadre des données statistiques qui devraient être ouvertes à la réutilisation libre et gratuite (Domange, 2013). Définies notamment par le Musée des Beaux-Arts de Toulouse et le collectif Savoirscomi, il s'agit de

[...] l'ensemble des données statistiques sur le fonctionnement et l'évaluation des opérateurs (budgets, statistiques de fonctionnements, de fréquentations, toutes les études et/ou enquêtes menées par les opérateurs ou financées par eux; les données des rapports externes commandés par le MCC, ainsi que les données des commissions relevant du MCC. (Domange, 2013, p. 7)

99

Force est de constater que les données ouvertes constituent aujourd'hui un ensemble sourcé à intégrer dans les corpus d'analyse statistique et une autre approche de la production de données. Néanmoins, elles restent encore pauvres, particulièrement concernant les musées, notamment : le Ministère de la Culture ne donne accès qu'à la liste des Musées de France, alors que les données du tourisme font déjà l'objet d'une interface plus développée chercheurs maîtrisant le langage de la programmation informatique. Les données ainsi exploitables doivent être entretenues, actualisées et leur utilisation

I. Voir le site www.datatourisme.gouv.fr 
nécessite intuition et clarté. Il s'agit donc d'une approche en développement, qui nécessitera de considérer dans le futur l'interopérabilité des données, aux normes internationales communes, et la mise en place d'une infrastructure adaptée (Mairesse, 2016).

\section{Repenser la production des données à l'âge du Big data ?}

Toujours dans un contexte de développement et de multiplication exponentielle des données numériques, les données massives (big data) se sont imposées dans la sphère publique et politique. Si elles créent de nouvelles opportunités commerciales pour le secteur privé, le champ investit également les universités, où les formations se se sont enrichies. Un tel développement interroge de fait la pertinence de ce phénomène pour la recherche, notamment en sciences sociales (Ollion \& Boelaert, 2015). Car elles provoquent une réflexion sur l'avenir des méthodes d'observations et sur les statistiques officielles, utilisées soit seules, soit associés à des sources de données traditionnelles (Conseil Economique et Social, 2013).

Cette typologie de données peut-elle transformer la manière d'étudier et de connaître? La réponse n'est pas aisée, notamment parce que ces données se définissent difficilement. On entend le Big data comme l'accumulation structurée ou non d'un gros volume de données aux sources très variées, caractérisées par leur masse, leur vélocité et leur diversité (les " $3 \mathrm{~V}$ ": volume, variété, vélocité), impliquant des formes novatrices de traitements pour améliorer leur connaissance et la prise de décision.

Les musées se sont emparés de cette opportunité en les exploitant en vue de l'accueil des publics et de l'amélioration de l'expérience de visite. Il s'agit par exemple des donnés issues des demandes d'informations, des inscriptions aux newsletters, des données issues des réseaux sociaux et du digital marketing, des opérations liées aux levées de fonds, des dispositifs de visite interactives, des systèmes de réservations, ou encore des programmes de fidélité. Le cas du Dallas Museum of Art est à ce titre précurseur : il capitalise depuis 20r3 sur ses données visiteurs pour développer une stratégie de développement soutenue par le Big data (Trendswatch, 2014) et la plateforme The DMA Friends permet de proposer aux visiteurs des contenus, en fonction de leur profil en temps réel. Aujourd'hui, la France opère cette transition au travers du projet Data $\&$ Musées, qui a pour but de réunir sur une plateforme unique et ouverte, les données des institutions culturelles, afin de développer des outils d'analyses et de prédiction pour les guider dans l'élaboration de stratégies et le développement de leurs activités. Le but des différents acteurs (le Centre des Monuments nationaux, Paris Musées, la société d'ingénierie culturelle Réciproque et la Chaire UNESCO-ITEN) est notamment de déduire des silhouettes-types de visiteurs et d'y associer des recommandations de contenus leur correspondant. L'utilisation de ces données présente donc un réel intérêt pour les institutions et pour les visiteurs, dans l'optique de fournir des analyses de la consommation 
des biens culturels, pour définir le positionnement des institutions ou proposer une personnalisation de la relation entre musée et visiteur.

On voit bien là l'usage commercial qui peut être faire au sein des institutions muséales. Ces données pourraient-elles investir le champ de la recherche? Se limitent-elles à la connaissance des pratiques des visiteurs ou peuvent-elles enrichir encore notre connaissance - en extension - du champ muséal ? D'après le CES, les données massives peuvent également produire des statistiques plus pertinentes et actuelles que les sources traditionnelles de la statistique officielle. C'est ce que tend à penser le groupe EGMUS, inaugurant ce thème en 2019 parmi les points stratégiques à investir pour le futur. Là encore cependant, les initiatives sont ténues, les problématiques encore mal maitrisées, et les politiques ne se sont pas encore emparés de ces enjeux. D'autant que ces données posent plusieurs problèmes, à la fois législatifs, financiers, mais aussi méthodologiques et technologiques, autant d'enjeux à résoudre et à anticiper pour accompagner le développement de la muséologie.

Finalement - et c'est certainement le point majeur pour le développement de la muséologie - ces enjeux d'avenir dépassent l'observation des tendances et vont engendrer des modifications structurelles du domaine de la recherche, notamment dans les observatoires. Dans un sens, l'apparition des données ouvertes ou massives - pose question concernant l'avenir des professionnels du secteur. L'augmentation toujours croissante des données engendre nécessairement l'intégration d'autres acteurs, notamment des sociétés d'audit et de conseils spécialisés dans la data, possédant une expertise en data science. A terme, cela va suppose également des besoins en formation pour la maîtrise de ces compétences particulières, notamment en programmation informatique. Le secteur professionnel lié aux observatoires et aux musées est amené à se renouveler Au travers de ces différentes tendances, ce n'est pas seulement la manière de penser l'observation qui est modifiée, mais bien les compétences nécessaires pour la développer.

\section{Conclusion : la muséologie au futur}

Si quelqu'un avait parlé ou écrit, il y a vingt ou trente ans, sur la muséologie comme science, il aurait été traité avec un sourire condescendant. Aujourd'hui la situation est fort différente. (Graesse, 1883, dans Mairesse, 2016)

Entre tradition et modernité, la muséologie évolue. Et dans cette réflexion sur son avenir, les observatoires de musées ont toute légitimité pour introduire ce champ de recherche. Longtemps mis à la marge des études en muséologie, pensés comme non essentiels, sauf à justifier l'existence et les subventions des institutions culturelles auprès des instances décisionnaires, l'observatoire et son 
domaine d'application permet d'aborder le champ muséal d'une manière plus globale, de réinterroger nos connaissances et surtout de mettre en évidence nos lacunes. D'avoir, finalement, la vision la plus complète - en positif ou en négatif - possible du phénomène muséal et de son évolution. Les questionnements et les méthodes que l'observatoire suppose, deviennent nécessaires pour savoir à quel monde nous sommes confrontés. Plusieurs réflexions seront à mener : sur leur constitution, sur leurs méthodes, sur la pertinence de leurs indicateurs, sur les transformations profondes - sociales et technologiques - du secteur, mais également sur la circulation des savoirs qu'ils induisent dans le cadre des société de l'information et du savoir. Ils ouvrent un véritable champ d'étude qui nécessite d'être pris en considération, pour permettre d'envisager des modèles de prédiction pour le futur des musées.

\section{Références}

Ambrose, T., \& Paine, C. (2012). Museums Basics. London: Routledge, ze éd.

Botte, J., Doyen, A., \& Uzlyte, L. (2017). « Ceci n'est pas un musée » : panorama géographique et historique des définitions du musée. Dans F. Mairesse (Dir.), Définir le musée du XXIe siècle (pp. I7-20). Paris : ICOFOM.

Brulon Soares, B. (2015). L'invention et la réinvention de la Nouvelle Muséologie. ICOFOM Study Series, 43a, 57-72.

Cardon, D. (2015). A quoi rêvent les algorithmes. Nos vies à l'heure des big data. Paris : Seuil.

Chevallier, D. (Dir.). (2013). Métamorphose des musées de société. Paris : La documentation française.

Conseil Economique et Social. (2013). Utilisation des «données massives » dans les statistiques officielles. Genève : ONU.

De Gruyter Saur. (2019). Museums of the world. Berlin, Boston : De Gruyter Saur, 26e éd.

De Varine, H. (2017). L'écomusée singulier et pluriel: un témoignage sur cinquante ans de muséologie communautaire dans le monde. Paris : L'Harmattan.

Dean, D., \& Edson, G. (1994). The handbook for museums. London : Routledge.

Debary, O. (2002). La fin du Creusot ou l'art d'accommoder les restes. Paris : Editions du CTHS.

Deloche, B. (2015). Pour une muséologie contractuelle. ICOFOM Study Series, $43 \mathrm{a}, 83-93$.

Desvallées, A., \& Mairesse, F. (Dir.). (20II). Dictionnaire encyclopédique de muséologie. Paris : Editions Armand Colin. 
Desvallées, A., \& Mairesse, F. (2005). Sur la muséologie. Culture \& Musées, 6, I3I-I55.

Domange, C. (Dir.). (2013). Rapport «Ouverture et partage des données culturelles publiques pour une (r)évolution numérique dans le secteur culturel ». Paris : Ministère de la culture, Département des programmes numériques du Secrétariat Général.

Eidelman, J. (Dir.). (20I7). Inventer les musées pour demain. Rapport de la mission Musées du XXIe siècle. Paris : Ministère de la Culture, La documentation française.

Georgel, C. (Dir). (I994). La jeunesse des musées : les musées de France au XIXe siècle. Paris : Réunion des musées nationaux.

Gob, A., \& Drouguet, N. (2010). La muséologie. Histoire, développements, enjeux, actuels. Paris, Editions Armand Colin.

Goode, G.B. (I895). The principles of museum administration. York : Coultas \& Volans.

Guibert, G., Rebillard, F., \& F. Rochelandet, F. (2016). Médias, culture et numérique. Approches socio-économiques. Paris : Armand Colin.

Frey, B., \& Meier, S. (2006). The Economics of Museums, Dans V. Ginsburg \& D. Throsby. Handbook of the Economics of Art and Culture. Amsterdam Elsevier, I, IOI7-I050.

Lehalle, E., \& Mironer, L. (I993). Musées et visiteurs : un observatoire permanent des publics. Paris : Ministère nationale de l'éducation et de la culture, Direction des musées de France, Département des publics, de l'action éducative et de la diffusion culturelle

Le Marec, J., \& Belaën, F. (2012). La création d'un observatoire : que s'agit-il de représenter? Communication \& Langages, I7I, 29-45.

Mairesse, F. (Dir.). (2016). Les nouvelles tendances de la muséologie. Paris : La documentation française.

Morishita, M. (2010). The Empty Museum. Western cultures and the Artistic Field in Modern Japan. Farnham: Ashgate.

Nuere, C.O. (Dir.). (20II). New challenges of cultural observatories. Bilbao: University of Deusto.

OEI-IBRAM. (20I6). Memory Spots: methodology and practices in social museology. OEI -Brasil: Phábrica.

Ollion, E., \& Boelaert, J. (2015). Au-delà des Big data. Sociologie, 3 ; 6.

Pomian, K. (1987). Collectionneurs, amateurs et curieux : Paris, Venise, XVIe-XVIIIe siécle. Paris : Gallimard. 
Pommier, E. (Dir.). (1995). Les musées en Europe à la veille de l'ouverture du Louvre : actes du colloque / organisé par le Service culturel du Musée du Louvre...les 3, 4 et 5 juin 1993. Paris : Louvre, Klincksieck,

Rivière, G-H. (1989). La muséologie selon Georges-Henri Rivière: cours de muséologie, textes et témoignages. Paris : Dunos

Sepulveda-Koptche, L. (2010). Les musées, leurs publics et les dynamiques sociales : l'approche de l'Observatoire des musées et centres culturels. Culture \& Musées, I6, I84-193.

Simon, N. (2010). The participatory museum. Santa-Cruz: Museum 2.o.

Zubiaur Carreño, F.J. (2004). Curso de museologia. Gijón : Tréa. 